\title{
Index rerum ad Vol. 158
}

\section{Confecit: C. Loeb-Schürch}

$(\mathrm{V})=$ Vortrag - Report - Communication

Ablatio, v. Fluoro-rétinographie Acuité visuelle, v. Arrestovisography,

Functions oculaires, Funktions-

prüfungen, Pleoptik Adaptation, v. Funktionsprüfungen,

Lichtsinn, Statische Perimetrie Aéronautique, v. Vols atmosphériques Agriculture; ocular hazards in agriculture, 125 (V) Alcool méthylique; intoxication par

Talcool méthylique, 141 (V) Allergie, v. Kontaktallergie Amblyopia, v. Arrestovisography,

Pleoptik Angiographie, v. Drug toxicity,

Fluoro-rétinographie, Frühdia-

gnose medikamentöser Schäden Angle camérulaire, v. Contusion Anoxie, v. Vols

atmosphériques Antibiotika, v. Infektionen Arbeitsmedizin, v. European Society

of Ophthalmology Argyrosis, v. Chemische Schädi-

gungen Arrestovisography in strabismic

amblyopia, 320 Aspergillus fumigatus, v. Agriculture Augenmuskeln, v. Fatigue visuelle,

Fractures de Torbite

Bakterielle Infektionen, v. Infektionen

Basedow'sche Krankheit, v. Endo-krine Ophthalmopathie

Berufskrankheiten, v. European Society of Ophthalmology

Bindehaut, v. Maladies oculaires externes

Binokularsehen, v. Polatest

Biochemie, v. Sonnenstrahlung

Bjerrum-Skotom, v. Glaukom-

Gesichtsfeld Blitz, v. Electrical injuries Blutdruck, v. Ophthalmodynamo-

metrie Brûlure, v. Injuries Burn, v. Injuries

Camera, v. Filmmikroskop

Cataract, v. Silicoextraction

Cavitation, v. Ultraschall

Chalkosis, v. Chemische Schädi-gungen

Chamber angle, v. Contusion

Champ visuel, v. Glaukom-Gesichts-feld, Hirntumoren, Kinetische Peri-metrie, Korrekte

Perimetrie, Lichtsinn, Röntgendiagnostik, Statische Perimetrie

Chelate, v. Chemische Schädigungen

Chemische Schädigungen des Auges in der Arbeitsmedizin, 95 (V)

Chirurgie, v. Extraction de corps étrangers, Fractures de Гorbite, Goniotomie, Interventions électro-magnétiques, Lider, Mikrochirur-gie, Silicoextraction, Wund-verschluss

Chloroquine, v. Drug toxicity, Früh-diagnose medikamentöser Schäden

Color sense, v. Funktionsprüfungen Statische Perimetrie 
Congrès, v. European Society of Ophthalmology, Schweizerische Ophthalmologische

Gesellschaft

Conjunctiva, v. Maladies oculaires externes

Consultations génétiques; les consultations génétiques en ophtalmo-logie, 521 (V)

Index rerum

ad Vol. 158

625

Contusio, v. Contusion, Injuries

Contusion of the eye, 49 (V)

Cornea, v. Electrical injuries, Maladies oculaires externes, Sonnenstrahlung, Threat

Corps étranger, v. Extraction de corps étrangers, Interventions électromagnétiques

Cours de perfectionnement; ouverture du cours de perfectionnement par le President de la SSO,

$354(\mathrm{~V})$

Cristallin, v. Electrical injuries, Extraction de corps étrangers, Human lens, Injuries, Threat, Vols atmosphériques

Décollement rétinien, v. Fluoro-rétinographie

Desinfektionsprobleme; aktuelle Sterilisations- und Desinfektionsprobleme in Klinik und Praxis unter besonderer Brücksichtigung der Ophthalmologie, 550 (V)

Development, v. Human lens

Diabetes, v. Fluoro-rétinographie, Microaneurysmata

Diagnostik, v. Fluoro-rétinographie, Frühdiagnose medikamentöser Schäden, Glaukom-

Gesichtsfeld, Hirntumoren, Lichtsinn, Ophthal-modynamometrie, Statische Peri-metrie

Diplopie, v. Fractures de $\Gamma$ orbite

Drug toxicity; some aspects of retinal drug toxicity, 232 (V)

Eclair, v. Electrical injuries

Effets secondaires, v. Drug toxicity,

Frühdiagnose medikamentöser

Schäden, Schädigungen des vorde-

ren Augenabschnittes, Threat Ekzem, v. Kontaktallergie Electrical injuries, 109 (V)

Electronystagmography, v. Arresto-

visography Elektromagnet, v. Extraction de

corps étrangers, Interventions

électromagnétiques Elektronenmikroskopie, v. Human

lens Elektrookulogramm, v. Drug toxicity Elektroretinogramm, v. Alcool

méthylique, Funktionsprüfungen

Embryologie, v. Human lens

Endokrine Ophthalmopathie; die endokrine Ophthalmopathied 565 (V)

Enophthalmus, v. Fractures de Гorbite

Entwicklung, v. Human lens

EOG, v. Drug toxicity

Epikutantest, v. Kontaktallergie

ERG, v. Alcool méthylique, Funktionsprüfungen

Ermüdung, v. Fatigue visuelle

European Society of Ophthalmology; 3rd Congress of the European Society of Ophthalmology, Amsterdam, 1968, 1-272 (V) 
Experimentelle Forschung, v. Microaneurysmata

Extraction de corps étrangers; Гex-traction de corps étrangers magné-tiques du cristallin transparent, $64(\mathrm{~V})$

Farbsinn, v. Funktionsprüfungen, Statische Perimetrie

Fatigue visuelle; la fatigue visuelle, 157 (V)

Filmmikroskop; das Zeiss-Film-mikroskop nach Barraquer, 310

Fixation excentrique, v. Pleoptik

Fliege, v. Agriculture

Flight, v. Vols atmosphériques

Flimmer-Perimetrie, v. Lichtsinn

Fluoroangiographie, v. Drug toxicity, Fluoro-rétinographie, Frühdiagnose medikamentöser

Schäden

Fluoro-rétinographie; Гinterêt pratique de la fluoro-rétinographie, 504 (V)

Fly, v. Agriculture

Fonctions oculaires; Гexamen des fonctions oculaires jusqu'à Гâge de 6 ans, 488 (V)

Foreign body, v. Extraction de corps étrangers, Interventions électromagnétiques

Fortbildung und Orientierung, 326

Fractures de Torbite; le traitement chirurgical des paralysies oculaires secondaires aux fractures de 1'orbite, 28 (V)

Frühdiagnose medikamentöser Schäden von Netzhaut und Seh-nerv, 245 (V)

626 Index rei

Funktionsprüfungen; Perimetrie und andere Funktionsprüfungen, 433 (V)

Further studies and other informations, 326

Gefässe, v. Fluoro-rétinographie, Hirntumoren, Micro-aneurysmata, Ophthalmodynamometrie,

Vols atmosphériques

Genetics, v. Consultations génétiques

Gesichtsfeld, v. Glaukom-Gesichtsfeld, Hirntumoren, Kinetische Perimetrie, Korrekte

Perimetrie, Lichtsinn, Röntgendiagnostik, Statische Perimetrie

Glaucoma, v. Glaukom-Gesichtsfeld, Vols atmosphériques

Glaukom-Gesichtsfeld, 469 (V)

Goggles, v. Prevention

Goniotomie; un dispositif simple et pratique pour la goniotomie, 292

Hérédité, v. Consultations génétiques Hirntumoren; Gesichtsfelddefekte bei

Hirntumoren, 451 (V) Histologie, v. Agriculture, Micro-aneurysmata, Sonnenstrahlung

Hornhaut, v. Electrical injuries, Maladies oculaires externes, Sonnenstrahlung, Threat

Hospitalismus, v. Desinfektions-

probleme Human lens; electron microscopic studies on the development of the human lens, 296 Hydrostatic capillary pressure,

v. Micro-aneurysmata Hydroxychloroquine, v. Drug toxicity Hyperthyreose, v. Endokrine Ophthalmopathie Hyphaema, v. Contusion Hypoderma bovis, v. Agriculture Hypoxie, v. Vols

atmosphériques

Infektionen; zur Therapie bakteriel-ler Infektionen des Auges, 326

Injuries; a retrospective study of eye injuries, 12 (V)

Instrument, v. Filmmikroskop, Goniotomie, Mikrochirurgie, Ophthalmodynamometrie, Silicoextraction, Spaltlampe

ad Vol. 158 
Interventions électromagnétiques

répétées, $57(\mathrm{~V})$

Intoxication, v. Alcool méthylique, Chemische Schädigungen

Intraokulardruck, v. Contusion, Threat

Intraokulare Infektionen, v. Infektionen

Intraokularer Fremdkörper, v. Extraction de corps étrangers, Interventions électromagnétiques

Irradiation solaire, v. Sonnenstrahlung

Kammerwinkel, v. Contusion Kaninchen, v. Micro-aneurysmata Katarakt, v. Silicoextraction

Kavitation, v. Ultraschall Keratomycosis, v. Agriculture Kinetische Perimetrie, 406 (V)

Kongresse, v. European Society of Ophthalmology, Schweizerische Ophthalmologische

Gesellschaft Kontaktallergie; Abklärung und Be-handlung von Kontaktallergien im Bereiche der

Augen, 540 (V) Korrekte Perimetrie; wichtige Be-dingungen für eine korrekte Perimetrie, 419

(V)

Lähmung, v. Fractures de Гorbite Lapin, v. Micro-aneurysmata Läppchenprobe, v.

Kontaktallergie Laserkoagulation, v. Lichtkoagulation Leber'sche Opticusatrophie, v.

Consultations génétiques Lens, v. Electrical injuries, Extraction de corps étrangers, Human lens,

Injuries, Threat, Vols atmosphériques Lesion, v. European Society of

Ophthalmology Licht, v. Sonnenstrahlung Lichtkoagulation; BeweГtung ver-schiedener

Verfahren der Lichtkoagulation, 601 (V) Lichtsinn mit besonderer Beriick-

sichtigung der Perimetrie, 362 (V) Lid, v. Kontaktallergie, Lider Lider; kleine Chirurgie der

Lider,

587 (V) Lightning, v. Electrical injuries

Index rer

Linse, v. Electrical injuries, Extraction de corps étrangers, Human lens, Injuries, Threat, Vols atmos-phériques

Lumière, $v$. Sonnenstrahlung

Macula-Läsionen, v. Fluoro-rétino-graphie

Magnetextraktor, v. Extraction de corps étrangers, Interventions électromagnétiques

Maladies oculaires externes par action directe d'éléments ex-térieurs, 181 (V)

Malformations, v. Consultations génétiques

Marfan-Syndrom, v. Consultations génétiques

Medikament, v. Drug toxicity, Früh-diagnose medikamentöser Schä-den, Kontaktallergie,

Schädigungen des vorderen Augenabschnittes, Threat

Métaux lourds, v. Chemische Schädigungen, Maladies oculaires externes

Micro-aneurysmata; retinal micro-aneurysmata, 273

Microscopie électronique, v. Human lens

Mikrochirurgie, 578 (V)

Mikroskop, v. Filmmikroskop

Missbildungen, v. Consultation génétiques

Mouche, v. Agriculture

Muscles oculaires, v. Fatigue visuelle, Fractures de Гorbite

Nebenwirkungen, v. Drug toxicity, Frühdiagnose medikamentöser Schäden, Schädigungen des vorderen Augenabschnittes, Threat

Nerf optique, v. Alcool méthylique, Consultations génétiques, Frühdiagnose medikamentöser Schäden 
Netzhaut, v. Alcool méthylique, Drug toxicity, Fluoro-rétino-graphie, Frühdiagnose medikamentöser Schäden, Micro-aneurysmata, Threat, Vols atmosphériques Neuroophthalmologie, v. Hirn-tumoren

627

urn ad Vol. 158

Nystagme opto-cinétique, v. Arresto-visography

Occupational and medicative hazards in ophthalmology, 1-272 (V)

Operation, v. Extractions de corps étrangers, Fractures de Гorbite, Goniotomie, Interventions électromagnétiques, Lider, Mikrochirurgie, Silicoextraction, Wundverschluss

Ophthalmodynamometrie, 342

Ophtalmologie; Гophtalmologie, sa position dans la médecine, au passé, au present et à $\Gamma$ avenir, 1 (V)

Ophthalmomyasis, v. Agriculture

Opticus, v. Alcool méthylique, Consultations génétiques, Frühdiagnose medikamentöser

Schäden

Optokinetic nystagmus, v. Arresto-visography

Orbitafrakturen, v. Fractures de Torbite

Palsy, v. Fractures de Torbite Papule, v. Fluoro-rétinographie Paralysie, v. Fractures de Гorbite

Paupière, v. Kontaktallergie, Lider Pédiatro-ophtalmologie, v. Fonctions

oculaires Perfectionnement et orientation, 326 Perforatio, v. Injuries Perimetrie, v. Glaukom-

Gesichtsfeld, Hirntumoren, Kinetische Perimetrie, Korrekte Perimetrie, Licht-sinn,

Röntgendiagnostik, Statische Perimetrie Pesticides; chemical burns due to

pesticides, 136 (V) Photokoagulation, v. Lichtkoagula-

tion, Micro-aneurysmata Physalaemin, v. Sjoegren's syndrome PigmentГetinopathie, v.

Consultations

génétiques Pilote, v. Vols atmosphériques Pleoptik; Sinn der Pleoptik, 334 (V) Polatest; die

Anwendung des Pola-

test in der Praxis, 612 (V) Pression hydrostatique intracapil-

laire, v. Micro-aneurysmata Pression oculaire, v. Contusion, Threat

628

Index rerum ad Vol. 158

Pression sanguine, v. Ophthalmo-

dynamometrie Prevention; the prevention of ocular

injuries in industry, 149 (V) Professional medicine, v. European

Society of Ophthalmology Profil-Perimetrie, v. Statische Peri-

metrie Protections oculaires, v. Prevention Protein, v. Sonnenstrahlung

Rabbit, v. Micro-aneurysmata

Radiation, v. Sonnenstrahlung

Recherches expérimentales, v. Micro-aneurysmata

Refraktion, v. Korrekte Perimetrie, Polatest

Retina, v. Alcool méthylique, Drug toxicity, Fluoro-rétinographie, Frühdiagnose

medikamentöser Schäden, Micro-aneurysmata, Threat, Vols atmosphériques

Retinitis pigmentosa, v. Consultations génétiques

Retinoblastom, v. Consultations génétiques 
Röntgendiagnostik und Gesichtsfeld, 611 (V)

Schädigungen des vorderen Augen-abschnittes durch Medikamente, 218 (V)

Schädlingsbekämpfungsmittel, v. Pesticides

Schielen, v. Arrestovisography, Pleoptik

Schutzbrillen, v. Prevention

Schwefel, v. Pesticides

Schweizerische Ophthalmologische Gesellschaft. 2. Fortbildungskurs, Bern, 1968, 354-623 (V)

Schwermetalle, v. Chemische Schädigungen, Maladies oculaires externes

Sclérose vasculaire, v. Fluoro-rétinographie

Sehschärfe, v. Arrestovisography, Functions oculaires, Funktions-prüfungen, Pleoptik

Side effects, v. Drug toxicity, Frühdiagnose medikamentöser Schäden, Schädigungen des

vorderen Augen-abschnittes, Threat

Siderosis, v. Chemische Schädigungen, Extraction de corps étrangers

Silicoextraction of senile and complicated cataracts, 288

Sjoegren's syndrome; the treatment of Sjoegren's syndrome with Physalaemin, 284

Société Suisse d'Ophtalmologie. 2e cours de perfectionnement, Berne, 1968, 354-623 (V)

Societies, v. European Society of Ophthalmology, Schweizerische Ophthalmologische

Gesellschaft

Sonnenstrahlung; ultraviolette und Sonnenstrahlung, 75 (V)

Soufre, v. Pesticides

Space flight, v. Vols atmosphériques

Spaltlampe; der neue Kreuzschlitten zur Haag-Streit-Spaltlampe, 622 (V)

Squint, v. Arrestovisography, Pleoptik

Statische Perimetrie; die Technik der statischen Perimetrie, 387 (V)

Sterilisation, v. Desinfektions-probleme

Strabismus, v. Arrestovisography, Pleoptik

Strahlen, v. Sonnenstrahlung

Sulphur, v. Pesticides

Surgery, v. Extraction de corps étrangers, Fractures de Гorbite, Goniotomie, Interventions

électro-magnétiques, Lider, Mikrochirurgie, Silicoextraction, Wundverschluss

Syndrome, v. Consultations génétiques, Sjoegren's syndrome

Tension oculaire, v. Contusion, Threat

Test, v. Fatigue visuelle

Therapie, v. Chemische Schädigungen, Drug toxicity, Extraction de corps étrangers, Fractures de Torbite, Infektionen, Injuries, Interventions électromagnétiques, Schädigungen des vorderen Augen-abschnittes, Sjoegren's syndrome, Threat

Threat; treating the patient a threat for his eyes, 208 (V)

Thrombose, v. Fluoro-rétinographie

Thyreoidea, v. Endokrine Ophthal-mopathie

Index rerum

ad Vol. 158

629

Trauma, v. European Society of

Ophthalmology Tumor, v. Consultations génétiques,

Fluoro-rétinographie, Hirntumoren 
Ulcus serpens, v. Infektionen Ultraschall; Schädigungen durch

Ultraschall am Auge, 118 (V) Ultraviolett-Strahlung, v. Sonnen-

strahlung Untersuchungsmethodik, v. Arresto-

visography, Fluoro-rétinographie,

korrekte Perimetrie, Lichtsinn,

Ophthalmodynamometrie

Vaisseaux, v. Fluoro-rétinographie, Hirntumoren, Micro-aneurysmata, Ophthalmodynamometrie,

Vols atmosphériques

Verbrennung, v. Injuries

Vererbung, v. Consultations génétiques

Vergiftung, v. Alcool méthylique,

Chemische Schädigungen Vessels, v. Fluoro-rétinographie,

Hirntumoren, Micro-aneurysmata,

Ophthalmodynamometrie, Vols

atmosphériques Visual acuity, v. Arrestovisography,

Functions oculaires, Funktions-

prüfungen, Pleoptik Visual fatigue, v. Fatigue visuelle Vols atmosphériques; maladies pro-

fessionnelles oculaires en rapport

avec les vols atmosphériques et

spatiaux, $191(\mathrm{~V})$

Weltraumflug, v. Vols atmosphériques

W. Wundverschluss; praktische Details für den Wundverschluss in der Augenchirurgie $590(\mathrm{~V})$

Xenonkoagulation

v. Lichtkoagu-lation 